\title{
POISSON INTEGRALS OF REGULAR FUNCTIONS
}

\author{
JOSÉ R. DORRONSORO
}

\begin{abstract}
Tangential convergence of Poisson integrals is proved for certain spaces of regular functions which contain the spaces of Bessel potentials of $L^{p}$ functions, $1<p<\infty$, and of functions in the local Hardy space $h^{1}$, and the corresponding tangential maximal functions are shown to be of strong $p$ type, $p \geq 1$.
\end{abstract}

1. Introduction. It is well known that for a general $L^{p}$ function $f, 1 \leq p \leq \infty$, its Poisson integral $u(x, y)=P_{y} * f(x)\left(P_{y}(z)=c_{n} y /\left(|z|^{2}+y^{2}\right)^{(n+1) / 2}, z \in \mathbf{R}^{n}, y>\right.$ 0 ) converges nontangentially to $f(x)$ a.e. when $y$ tends to 0 . It is also well known $\left[18\right.$, p. 280] that for general $L^{p}$ functions this result fails when convergence inside regions with some degree of tangentiality is considered.

However, tangential convergence holds for certain classes of functions: Nagel, Rudin, and Shapiro have recently established [14] the existence of tangential limits for a large class of potentials of $L^{p}$ functions (see also [14] for earlier results). A particular instance are the spaces $L_{a}^{p}=\left\{J_{a} * f: f \in L^{p}\right\}, 1 \leq p \leq \infty,\left(J_{a}\right)^{\wedge}(z)=$ $\left(1+|z|^{2}\right)^{-a / 2}$, of Bessel potentials of $L^{p}$ functions, for which explicit approach regions are given: if $1 \leq p \leq n / a$ and $x \in \mathbf{R}^{n}$, define $D_{a, p}(x)$ as

(i) $D_{a, p}(x)=\left\{(z, y) \in \mathbf{R}_{+}^{n+1}:|z-x| \leq y^{1-a p / n}\right\}, p<n / a$, 1 ,

(ii) $D_{a, p}(x)=\left\{(z, y) \in \mathbf{R}_{+}^{n+1}:|z-x| \leq(\log 1 / y)^{-(p-1) / n}, y \leq 1 / e\right\}, p=n / a>$

(iii) $D_{n, 1}(x)=\left\{(x, y) \in \mathbf{R}_{+}^{n+1}:|z-x| \leq(\log 1 / y)^{1 / n}, y \leq 1 / e\right\}$.

Then [14, Theorems 2.9, 3.13, and 5.5]

(i) if $1 \leq p \leq n / a$ and $f \in L_{a}^{p}, u(x, y)=P_{y} * f(z)$ tends to $f(x)$ inside $D_{a, p}(x)$ for a.e. $x \in \mathbf{R}^{n}$;

(ii) if $1<p \leq n / a, f \in L_{a}^{p}$ and $0<b<a, u(z, y)$ tends to $f(x)$ inside $D_{b, p}(x)$ for $B_{a-b, p}$ a.e. $x \in \mathbf{R}^{n}\left(B_{s, t}\right.$ denotes $(s, t)$ Bessel capacity; see $\left.\S 2\right)$.

Note that if $a>n / p$ and $f \in L_{a}^{p}, f$ is continuous.

Furthermore, it is shown in [14, Theorem 3.8] that the corresponding maximal operators $T_{a, p} f(x)=\sup \left\{|u(z, y)|:(z, y) \in D_{a, p}(x)\right\}$ verify $\left\|T_{a, p} f\right\|_{p} \leq C\|f\|_{L_{a}^{p}}$, whereas for $p=1$ Nagel and Stein proved [15, Theorem 5] that if $F$ is in the Hardy space $H^{1},\left\|T_{a, 1}\left(J_{a} F\right)\right\|_{1} \leq C\|F\|_{H^{1}}, a<n([15]$ also contains results for Bessel potentials of $\left.H^{p}, p>0\right)$.

The tangentiality of the approaching regions is shown in [14] to depend on the corresponding Bessel kernels $J_{a}$; here we will see how it can also be related to the regularity of the $L_{a}^{p}$ functions. In fact, similar results (Theorems 1 and 2 below) hold for a larger class of functions, which we now define. If $\mathbf{P}_{k}$ denotes the set of

Received by the editors July 17,1985 .

1980 Mathematics Subject Classification (1985 Revision). Primary 31B25; Secondary 46E35.

Partially supported by C.A.I.C.Y.T. 2805-83. 
all polynomials of degree $k, x \in \mathbf{R}^{n}, t>0,1 \leq r \leq \infty$ and $f \in L_{\text {loc }}^{1}$, consider the "polynomial approximation" operator

$$
E_{r}^{k} f(x, t)=\sup \inf _{P \in \mathbf{P}_{k}}\left(f_{Q}|f-P|^{r}\right)^{1 / r}
$$

the sup taken over all cubes $Q$ with $x \in Q$ and having Lebesgue measure $|Q|=t^{n}$ (throughout the paper $f_{E} f$ or $f_{E}$ stand for the mean $\int_{E} f d z /|E|$ ).

Now, if $a>0$ and $m=[a]$, its integral part, we define $G_{a} f(x, t)=$ $\sup _{s \leq t} s^{-a} E_{1}^{m} f(x, s), G_{a} f(x)=G_{a} f(x, \infty)$ (in what follows, if $k=m$ and $r=1$, we will write $E f(x, t)$ instead of $\left.E_{1}^{m} f(x, t)\right)$; then $C_{a}^{p}, 1 \leq p \leq \infty$, denotes the space of those $L^{p}$ functions $f$ such that $G_{a} f \in L^{p}$; with the norm $\|f\|_{a, p}=\|f\|_{p}+\left\|G_{a} f\right\|_{p}$, $C_{a}^{p}$ becomes a Banach space. These spaces were introduced by Calderón and Scott [4] and are extensively studied by Devore and Sharpley in [8].

Our results are given for a proper subset of $C_{a}^{p}$, the closed subspace $F_{a}^{p}$ of those $f \in C_{a}^{p}$ such that $G_{a} f(x, t)=o(1)$ a.e. as $t$ goes to 0 (in fact $F_{a}^{p}, p<\infty$, is the closure of $C_{0}^{\infty}$, the compactly supported $C^{\infty}$ functions; see $\S 3$ ). $C_{a}^{p}$ and $F_{a}^{p}$ can be seen as global versions of the spaces $T_{a}^{p}(x)$ and $t_{a}^{p}(x)$ of Calderón and Zygmund [5]. If $1<p<\infty, L_{a}^{p}$ is continuously imbedded in $F_{a}^{p}$; indeed, $f \in L_{a}^{p}$ iff $f \in L^{p}$ and

$$
G_{a, 2} f(x)=\left(\int_{0}^{\infty} E f(x, t)^{2} t^{-2 a-1} d t\right)^{1 / 2} \in L^{p},
$$

and $\|f\|_{L_{a}^{p}} \sim\|f\|_{p}+\left\|G_{a, 2} f\right\|_{p}$ (see [9]; by $A \sim B$ we mean that $A / C \leq B \leq C A$, for some constant $C$; in what follows $C$ will stand for any constant independent of sets, points, or functions, and not necessarily the same on each appearance). However, although the imbedding $L_{a}^{p} \subset F_{a}^{p}$ is proper, the Poisson integrals of functions in $F_{a}^{p}$ and $L_{a}^{p}$ have the same tangential behavior:

THEOREM 1. If $1 \leq p<n / a$ or $p=n / a>1$ and $f \in F_{a}^{p}$, then $u(z, y)=$ $P_{y} * f(z)$ tends to $f(x)$ a.e. when $(z, y)$ tends to $x$ inside $D_{a, p}(x)$.

The restriction $p \leq n / a$ is due to the fact that functions in $F_{a}^{p}$ are continuous when $p>n / a$, and the same is true in $F_{n}^{1}[8$, p. 68].

For functions in $F_{a}^{p}$ the exceptional set also becomes smaller when the tangentiality of the approach regions is decreased; in fact the results of [14] can be slightly improved:

THEOREM 2. (i) If $f \in F_{a}^{p}, 1 \leq p<n / a$, and $0<b<a$, then $u(z, y)$ converges to $f(x)$ inside $D_{b, p}(x)$ for all $x$ except a set of zero $H^{n-(a-b) p}$ Hausdorff measure; if moreover $p>1$, $u$ converges nontangentially to $f(x) B_{a, p}$-a.e.

(ii) If $p=n / a>1$ and $p<r<\infty, u$ converges to $f(x)$ inside $D_{n / r, r}(x)$ for $H^{n p / r}$-a.a. $x$, whereas if $b$ is such that $0 \leq b<n / p, u$ converges to $f(x)$ inside $D_{b, p}(x)$ for $B_{n / p, p}-a . a . x$.

Theorem 2 requires some explanation: functions in $F_{a}^{p}$ are defined in principle only a.e.; Theorem 2 will be shown to hold after suitably redefining them on a zero measure set.

As could be expected, Theorems 1 and 2 are deduced from weak type estimates for the tangential maximal operators $T_{a, p} f(x)=\sup \left\{|u(z, y)|:(z, y) \in D_{a, p}(x)\right\}$, but since functions in $F_{a}^{p}$ are not representable as potentials of $L^{p}$ functions, we rely 
on certain Sobolev and Trudinger type inequalities for them (Theorem 5). However, these weak type inequalities can be strengthened.

THEOREM 3. If $f \in C_{a}^{p}, 1 \leq p \leq n / a$, then $\left\|T_{a, p} f\right\|_{p} \leq C\|f\|_{a, p}$.

The proof of Theorem 3 is modelled after that of Theorem 3.8 in [14], but with an important difference: the key argument in [14], Hansson's strong capacitary estimates [10], is no longer available here and a strong estimate, valid if $1 \leq p<\infty$, for a certain $C_{a}^{p}$ capacity type function, is proved (Theorem 6) along the lines of similar results by Adams [2] and Dahlberg [7].

Besides $L_{a}^{p}$, the so-called Triebel-Lizorkin spaces $F_{a}^{p, q}, 1 \leq p, q<\infty, a>0$ (see [17] or $\S 6$ for the definition) are also continuously imbedded in $F_{a}^{p}$ (Proposition 3 ) and therefore, the above theorems apply to them; we point out that if $1<p<\infty$, $F_{a}^{p, 2}=L_{a}^{p}$, whereas $F_{a}^{1,2}$ coincides with the space of Bessel potentials of functions in D. Goldberg's local Hardy space $h^{\mathbf{1}}[\mathbf{1 7}$, p. 51]. We also remark that Y. Mizuta has recently proved [13] results similar to those of Theorems 1 and 2 for functions being locally in the Besov space $B_{a}^{p, p}, 0<a<1$. Since $B_{a}^{p, p}=F_{a}^{p, p}$, Theorems 1 and 2 contain a global version of Mizuta's results.

The paper is organized as follows: $\S 2$ contains certain preliminary facts about capacities and Hausdorff measures. The spaces $F_{a}^{p}$ are studied in some detail in $\S 3$. Theorems 1 and 2 are proved in $\S 4$ and Theorem 3 in $\S 5$. Finally, in $\S 6$ TriebelLizorkin spaces $F_{a}^{p, q}, 1 \leq p, q<\infty, a>0$ are considered.

2. Preliminary results. For $a>0 J_{a}$ will denote the Bessel kernel of order $a$, $\left(J_{a}\right)^{\wedge}(z)=\left(1+|z|^{2}\right)^{-a / 2}$, and $I_{a}$ the Riesz kernel, $I_{a}(z)=c_{n, a}|z|^{a-n}, 0<a<n$; we will also denote by $J_{a}$ and $I_{a}$ the corresponding potential operators. The Bessel capacity $B_{a, p}$ and the Riesz capacity $R_{a, p}$ are defined for $E \subset \mathbf{R}^{n}$ as

$$
\begin{array}{ll}
B_{a, p}(E)=\inf \left\{\|f\|_{p}^{p}: f \geq 0, J_{a} f \geq \chi_{E}\right\}, & a>0, \\
R_{a, p}(E)=\inf \left\{\|f\|_{p}^{p}: f \geq 0, I_{a} f \geq \chi_{E}\right\}, & 0<a<n / p
\end{array}
$$

$\left(\chi_{E}=\right.$ characteristic function of $\left.E\right)$. If $a<n / p$,

$$
R_{a, p}(E) \leq B_{a, p}(E) \leq C\left(R_{a, p}(E)+R_{a, p}(E)^{n / n-a p}\right)
$$

[1]; thus, both have the same zero sets (see [12] for more properties of $R_{a, p}$ and $\left.B_{a, p}\right)$.

If $f \in L^{p}$ we obviously have

$$
\begin{gathered}
R_{a, p}\left(\left\{\left|I_{a} f\right|>t\right\}\right) \leq\left(\|f\|_{p} / t\right)^{p}, \quad 0<a<n / p \\
B_{a, p}\left(\left\{\left|J_{a} f\right|>t\right\}\right) \leq\left(\|f\|_{p} / t\right)^{p}
\end{gathered}
$$

thus, if $M f$ denotes the Hardy-Littlewood maximal operator, $M f(x)=\sup \left\{|f|_{Q}\right.$ : $x \in Q\},(1),(2)$ and the obvious inequalities $M\left(I_{a} f\right) \leq I_{a}(M f), M\left(J_{a} f\right) \leq J_{a}(M f)$ imply that the complements of the Lebesgue sets of $I_{a} f$ and $J_{a} f$ have zero $R_{a, p}$ and $B_{a, p}$ capacity respectively.

Related to $B_{a, p}$ and $R_{a, p}$ is the $H^{n-a p}$ Hausdorff measure: if $0<r \leq \infty$ and $E \subset \mathbf{R}^{n}$ we define

$$
H_{r}^{n-a p}(E)=\inf \left\{\sum_{0}^{\infty}\left|Q_{i}\right|^{1-a p / n}\right\}
$$


the inf taken over all coverings of $E$ by cubes of side $\leq r$; then $H^{n-a p}(E)=$ $\sup _{r} H_{r}^{n-a p}(E) . H^{n-a p}$ is finer than $B_{a, p}$ in the sense that $B_{a, p}(E) \leq C H_{\infty}^{n-a p}(E)$ [12]. Here we shall use $H_{\infty}^{n-a p}$ rather than $H^{n-a p}$; both have the same zero sets $[6]$.

If $0<a<n, 1 \leq p<n / a$, and $f \in L^{p}$, we define

$$
M_{a} f(x)=\sup \left\{|Q|^{a / n}|f|_{Q}: x \in Q\right\} .
$$

LEMMA 1. For the above $a, p$, and $f, H_{\infty}^{n-a p}\left(\left\{M_{a} f>t\right\}\right) \leq C\left(\|f\|_{p} / t\right)^{p}$.

PROOF. For each $x \in E=\left\{M_{a} f>t\right\}$ there is a cube $Q$ with $x \in Q$ and

$$
t<|Q|^{a / n}|f|_{Q} \leq|Q|^{a / n-1 / p}\left(\int_{Q}|f|^{p}\right)^{1 / p} ;
$$

hence, selecting $\left[\mathbf{1 6}\right.$, p. 9] a disjoint family $\left\{Q_{i}\right\}$ such that $E \subset \bigcup 5 Q_{i}(r Q$ denotes the cube with same center as $Q$ and side $r$ times side $(Q)$ ), we have

$$
H_{\infty}^{n-a p}(E) \leq C \sum\left|Q_{i}\right|^{1-a p / n} \leq C t^{-p} \sum \int_{Q_{i}}|f|^{p} \leq C\left(\|f\|_{p} / t\right)^{p} .
$$

Obviously, the same estimate holds with $M_{a}$ replaced by $\left(M_{a s}|f|^{s}\right)^{1 / s}, 1<$ $s \leq p$. Also, if we define for $0<r \leq 1 / 100$ and $\varphi(t)=(\log 1 / t)^{1-p}, H_{r}^{\varphi}(E)=$ $\inf \left\{\sum \varphi\left(\left|Q_{i}\right|\right): E \subset \cup Q_{i}, Q_{i}\right.$ cubes, side $\left.Q_{i} \leq r\right\}$ and the maximal operator $M_{\varphi} g(x)=\sup \left\{\int_{Q}|g| / \varphi(|Q|): x \in Q\right.$, side $\left.Q \leq 1 / 1000\right\}$, the above argument gives the estimate

$$
H_{1 / 100}^{\varphi}\left(\left\{M_{\varphi} g>t\right\}\right) \leq C\|g\|_{1} / t .
$$

LEMMA 2. If $0<b \leq a<n, 1 \leq p<n / a$ and $f \in L^{p}$, then

$$
H_{\infty}^{n-(a-b) p}\left(\left\{I_{a} f>t\right\}\right) \leq C\left(\|f\|_{p} / t\right)^{p(n-(a-b) p) /(n-a p)} .
$$

PROOF. The desired inequality follows from Lemma 1 once we prove

$$
\left|I_{a} f(x)\right| \leq C\|f\|_{p}^{b p /(n-(a-b) p)} M_{a-b} f(x)^{1-b p /(n-(a-b) p)} ;
$$

now, as in [11, Theorem 1], we have for any $r>0$

$$
\begin{aligned}
\left|I_{a} f(x)\right| & \leq C\left(\int_{|z| \leq r}+\int_{|z|>r}\right)|f(x-z)||z|^{a-n} d z \\
& \leq C \sum_{0}^{\infty}\left(2^{-k} r\right)^{a-n} \int_{|z| \leq 2^{-k_{r}}}|f(x+z)| d z+C r^{a-n / p}\|f\|_{p} \\
& \leq C\left(r^{b} M_{a-b} f(x)+r^{a-n / p}\|f\|_{p}\right)
\end{aligned}
$$

and (3) follows if we choose $r=\left(M_{a-b} f(x) /\|f\|_{p}\right)^{1 /(a-b-n / p)}$

LEMMA 3. There is a constant $C_{I}$ such that $M\left(I_{a} f\right) \leq C_{I} I_{a} f$ for all positive f. Also, there is a $C_{J}$ such that $f_{Q} J_{a} f(x+z) d z \leq C_{J} J_{a} f(x)$ for all cubes $Q$ centered at 0 with side $\leq 10$ and all $f \geq 0$.

PROOF. If $Q$ has center 0 , an easy computation gives $f_{Q} I_{a}(x+z) d z \leq C_{I} I_{a}(x)$; if moreover side $(Q) \leq 10, f_{Q} J_{a}(x+z) d z \leq C_{J} J_{a}(x)[\mathbf{3}, \mathrm{p}$. 418]. The lemma now follows.

As a consequence, if $g \geq 0$ and $f=J_{a} g, m f(x) \leq C f(x)$, where $m$ denotes the "local" maximal operator $m f(x)=\sup \left\{|f|_{Q}: x \in Q,|Q| \leq 5^{n}\right\}$. 
3. The spaces $F_{a}^{p}$. We fix $a>0, m=[a]$ and $p$ such that $1 \leq p \leq \infty$. We first show that $E f$ can be defined using a minimizing polynomial on each cube $Q$; in fact, if $P_{Q} f$ denotes the unique polynomial in $\mathbf{P}_{m}$ such that for any $\gamma=\left(\gamma_{1}, \ldots, \gamma_{n}\right) \in \mathbf{N}^{n}$ with $|\gamma|=\gamma_{1}+\cdots+\gamma_{n} \leq m$,

$$
\int_{Q}\left(f(y)-P_{Q} f(y)\right) y^{\gamma} d y=0
$$

then $[8$, p. 17]

$$
\text { if } D^{\gamma}=\left(\partial / \partial x_{1}\right)^{\gamma_{1}} \cdots\left(\partial / \partial x_{n}\right)^{\gamma_{n}}, \underset{Q}{\operatorname{ess} s u p}\left|D^{\gamma} P_{Q} f\right| \leq C|Q|^{-|\gamma| / n}|f|_{Q}
$$

it now follows that

$$
\text { for any } R \in \mathbf{P}_{k}, \quad f_{Q}\left|f-P_{Q} f\right| \leq C f_{Q}|f-R|,
$$

and therefore, $E f(x, t) \sim \sup \left\{f_{Q}\left|f-P_{Q} f\right|: x \in Q,|Q|=t^{n}\right\}$;

$$
\text { if } Q \subset Q^{\prime}, \quad f_{Q}\left|f-P_{Q} f\right| \leq C\left(\left|Q^{\prime}\right| /|Q|\right) f_{Q^{\prime}}\left|f-P_{Q^{\prime}} f\right| \text {; }
$$

in particular, if $Q_{x, t}$ denotes the cube with center $x$ and side $t$,

$$
G_{a} f(x) \sim \sup _{t>0} t^{-a} f_{Q_{x, t}}\left|f-P_{Q_{x, t}} f\right|
$$

also, balls can be used instead of cubes to define $E f$ and $G_{a} f$.

Fix next $x \in Q,|Q|=t^{n}$ and let $Q_{1} \subset Q_{2} \subset \cdots \subset Q_{k}=Q$ be a sequence of cubes with $x \in Q_{1}$ and $\left|Q_{i+1}\right|=2^{n}\left|Q_{i}\right|, i=1, \ldots, k-1$; writing the polynomials $P_{Q_{i}} f$ as $P_{Q_{i}} f(y)=\sum_{|\gamma| \leq m} c_{\gamma}\left(Q_{i}\right)(y-x)^{\gamma} / \gamma !$, we have by (4)

$$
\begin{aligned}
\left|c_{\gamma}\left(Q_{1}\right)-c_{\gamma}(Q)\right| & \leq \sum_{1}^{k-1}\left|c_{\gamma}\left(Q_{i}\right)-c_{\gamma}\left(Q_{i+1}\right)\right| \\
& \leq \sum\left|D^{\gamma}\left(P_{Q_{i}} f-P_{Q_{i+1}} f\right)(x)\right| \\
& \leq C \sum\left(2^{-i} t\right)^{-|\gamma|} E f\left(x, 2^{-i} t\right) \\
& \leq C \int_{2^{-k} t}^{t} E f(x, s) s^{-|\gamma|-1} d s ;
\end{aligned}
$$

in particular, since $P_{Q} f(x)=c_{0}(Q)$ tends to $f(x)$ a.e. [8, p. 9], we have

$$
\left|f(x)-c_{0}(Q)\right|=\left|f(x)-P_{Q} f(x)\right| \leq C \int_{0}^{t} E f(x, s) d s / s .
$$

Next, $C_{a}^{p}=\left\{f \in L^{p}:\|f\|_{a, p}=\|f\|_{p}+\left\|G_{a} f\right\|_{p}\right\}$ is a Banach space [8, p. 37] and $F_{a}^{p}=\left\{f \in C_{a}^{p}: G_{a} f(x, t)=o(1)\right\}$ can also be defined as the subspace of those $f \in C_{a}^{p}$ such that $\left\|G_{a} f(\cdot, t)\right\|_{p}=o(1)$ : indeed, since $G_{a} f(x, t) \leq G_{a} f(x)$, if $f \in F_{a}^{p}$, $\left\|G_{a} f(\cdot, t)\right\|_{p}=o(1)$ by dominated convergence; conversely, $\left\|G_{a} f(\cdot, t)\right\|_{p}=o(1)$ implies that $G_{a} f\left(x, t_{j}\right)=o(1)$ for some subsequence $t_{j}$, but then $f \in F_{a}^{p}$, for $G_{a} f(x, t) \leq G_{a} f\left(x, t_{j}\right)$ if $t \leq t_{j}$. Furthermore, it can be easily checked that $F_{a}^{p}$ is a closed subspace of $C_{a}^{p}$. 
Also, if $a$ is not an integer and $f \in C_{a}^{p}$, for a.e. $x$ there is a polynomial $P_{x} f \in \mathbf{P}_{m}$ such that $[8$, p. 32]

$$
C^{\prime} G_{a} f(x, t) \leq S_{a} f(x, t)=\sup _{s \leq t} s^{-a} f_{Q_{x, s}}\left|f-P_{x} f\right| \leq C G_{a} f(x, t)
$$

if $0<a<1, P_{x} f$ is the constant polynomial $f(x)$. Furthermore, setting $\chi_{t}=$ $t^{-n} \chi_{Q_{0, t}},(4)$ gives for $t \geq 1$

$$
t^{-a} E f(x, t) \leq C \int_{1}^{\infty}|f| * \chi_{s}(x) s^{-a-1} d s
$$

and therefore,

$$
\left\|\sup _{t \geq 1} t^{-a} E f(x, t)\right\|_{p} \leq C \int_{1}^{\infty}\left\||f| * \chi_{s}\right\|_{p} s^{-a-1} d s \leq C\|f\|_{p} ;
$$

as a consequence, $\|f\|_{a, p} \sim\|f\|_{p}+\left\|G_{a}(\cdot, 1)\right\|_{p}$.

THEOREM 4. For all positive $a$ and $b, J_{b}$ is an isomorphism from $C_{a}^{p}$ and $F_{a}^{p}$ onto $C_{a+b}^{p}$ and $F_{a+b}^{p}$ respectively; that is, if $f \in F_{a+b}^{p}\left(C_{a+b}^{p}\right)$ there is a unique $g \in F_{a}^{p}\left(C_{a}^{p}\right)$ such that $f=J_{b} g$ and $\|f\|_{a+b, p} \sim\|g\|_{a, p}$.

PROOF. Assuming $b<n$ (the general case follows by the semigroup property of $J$ ) we show first that $\left\|J_{b} f\right\|_{a+b, p} \leq C\|f\|_{a, p}$. Fix $x \in \mathbf{R}^{n}$ and $Q$ with $x \in Q$, $|Q|=t^{n}$; if $T(u, v)=\sum_{|\gamma| \leq p} D^{\gamma} J_{b}(u) v^{\gamma} / \gamma$ ! denotes the Taylor polynomial of degree $p=[a+b]$ of $J_{b}$ at $u$, consider the polynomial in $y$

$$
R_{Q}(y)=P_{Q} f * J_{b}(y)+\int_{c_{2 Q}}\left(f(z)-P_{Q} f(z)\right) T(x-z, y-x) d z
$$

since $\left|D^{\gamma} J_{b}(u)\right| \leq C\left(1+|u|^{b-n-|\gamma|}\right) e^{-|u|}\left[\mathbf{5}\right.$, p. 192], $R_{Q}$ is well defined and

$$
\begin{aligned}
\left|J_{b} f(y)-R_{Q}(y)\right| \leq & \int_{2 Q}\left|f(z)-P_{Q} f(z)\right| J_{b}(y-z) d z \\
& +\int_{c_{2 Q}}\left|f(z)-P_{Q} f(z)\right|\left|J_{b}(y-z)-T(x-z, y-x)\right| d z \\
= & \text { I }+ \text { II. }
\end{aligned}
$$

Clearly,

$$
f_{Q} I d y \leq \int_{2 Q}\left|f(z)-P_{Q} f(z)\right| f_{Q} J_{b}(y-z) d y \leq C t^{b} E f(x, 2 t),
$$

and by Taylor's formula and the fact that $|x-z+\theta(y-x)| \geq|x-z| / 2$ if $\theta \leq 1$ and $|x-z| \geq 2|x-y|$,

$$
\begin{aligned}
\mathrm{II} & \leq C t^{p+1} \int_{c_{2 Q}}\left|f(z)-P_{Q} f(z)\right| \cdot|x-z|^{b-n-p-1} d z \\
& \leq C t^{p+1} \sum_{0}^{\infty}\left(2^{k} t\right)^{b-m-1}\left(E f\left(x, 2^{k} t\right)+\underset{2^{k} Q}{\operatorname{ess} \sup }\left|P_{2^{k} Q} f-P_{Q} f\right|\right)
\end{aligned}
$$


writing $P_{2^{k} Q} f(z)=\sum_{|\gamma| \leq m} c_{\gamma}\left(2^{k} Q\right)(z-x)^{\gamma} / \gamma !,(7)$ gives for $z \in 2^{k} Q$

$$
\left|P_{2^{k}} f(z)-P_{Q} f(z)\right| \leq C \sum_{0}^{m}\left(2^{k} t\right)^{j} \int_{t}^{2^{k} t} E f(x, s) s^{-j-1} d s,
$$

which, since $a+b-p-1<0$, implies by Fubini's theorem

$$
\begin{aligned}
\mathrm{II} & \leq C t^{p+1}\left(\int_{t}^{\infty} s^{b-p-1}\left(E f(x, s)+\sum_{0}^{m} s^{j} \int_{t}^{s} E f(x, u) u^{-j-1} d u\right) d s / s\right) \\
& \leq C t^{p+1} \int_{t}^{\infty} s^{b-p-1} E f(x, s) d s / s .
\end{aligned}
$$

Now, putting (9) and (10) together,

$$
\begin{aligned}
E J_{b} f(x, t) & \leq C\left(t^{b} E f(x, 2 t)+t^{p+1} \int_{t}^{\infty} s^{b-p-1} E f(x, s) d s / s\right) \\
& \leq C t^{a+b} G_{a} f(x),
\end{aligned}
$$

and thus, $\left\|J_{b} f\right\|_{a+b, p} \leq C\|f\|_{a, p}$. Also, if $f \in F_{a}^{p}$, given $\varepsilon>0$ and $T$ such that $G_{a} f(x, T) \leq \varepsilon$,

$$
\begin{aligned}
t^{-a-b} E J_{b} f(x, t) & \leq C\left(\varepsilon+t^{p+1-a-b}\left(\int_{t}^{T}+\int_{T}^{\infty}\right)\left(s^{b-p-1} E f(x, s) d s / s\right)\right) \\
& \leq C\left(\varepsilon+(t / T)^{p+1-a-b} G_{b} f(x)\right) \leq C \varepsilon
\end{aligned}
$$

if $t$ is small enough; hence $J_{b} f \in F_{a+b}^{p}$.

Next, if $f \in F_{a}^{p}, a>1$, its weak partials $f_{i}=\partial f / \partial x_{i}$ verify $\left\|G_{a-1} f_{i}\right\|_{p} \leq$ $C\left\|G_{a} f\right\|_{p}\left[\mathbf{8}\right.$, p. 42], and also $\left|f_{i}(x)\right| \leq C\left(G_{a} f(x)+|f|_{Q_{x, 1}}\right)$ and

$$
E f_{i}(x, t) \leq C\left(\int_{0}^{t} M(E f(\cdot, s))(x) s^{-2} d s+E f(x, 2 t) / t\right)
$$

$\left[9\right.$, Theorem 3 and Lemma 1]; hence $f_{i} \in F_{a-1}^{p}$. This and the obvious imbeddings $F_{a}^{p} \subset F_{a-\varepsilon}^{p}$ imply that $I-\Delta$ maps $F_{a}^{p}, a>2$, into $F_{a-2}^{p}$ and $\|(I-\Delta) f\|_{a-2, p} \leq$ $C\|f\|_{a, p}$. Therefore, if $0<b<2$ and $f \in F_{a+b}^{p}, a>0, f=J_{b}(I-\Delta) J_{2-b} f=J_{b} g$, where $g \in F_{a}^{p}$ and $\|f\|_{a+\dot{b}, p} \sim\|g\|_{a, p}$. The same argument works for the $C_{a}^{p}$ and for a general $b>0$. The theorem follows by the semigroup properties of $J$.

Proposition 1. $C_{0}^{\infty}$ is dense in $F_{a}^{p}, 1 \leq p<\infty$.

ProOF. Supposing first $0<a<1$, let $\varphi \geq 0$ be a $C^{\infty}$ function with $\varphi(x)=1$ when $|x| \leq 1 / 10, \varphi(x)=0$ when $|x| \geq 1$ and $\int \varphi d x=1$, and set $\varphi_{r}(x)=$ $r^{-n} \varphi(x / r), r>0$. If $f \in F_{a}^{p}$ and $f_{r}=f * \varphi_{r}(x)$, an easy computation yields $G_{a} f_{r}(x, t) \leq C \varphi_{r} * G_{a} f(\cdot, t)(x)$. Thus, given $\varepsilon$, if $\left\|G_{a} f(\cdot, T)\right\|_{p} \leq \varepsilon$ and $r$ is small enough, (4) implies

$$
\begin{aligned}
\left\|G_{a}\left(f-f_{r}\right)\right\|_{p} & \leq C\left\|G_{a} f(\cdot, T)\right\|_{p}+C\left\|\int_{T}^{\infty} t^{-a}\left|f-f_{r}\right| * \chi_{t}(\cdot) d t / t\right\|_{p} \\
& \leq C\left\|G_{a} f(\cdot, T)\right\|_{p}+C \int_{T}^{\infty}\left\|f-f_{r}\right\|_{p} t^{-a-1} d t \\
& \leq C \varepsilon+C T^{-a}\left\|f-f_{r}\right\|_{p} \leq C \varepsilon .
\end{aligned}
$$


Next, setting $\varphi^{r}(x)=\varphi(r x)$ and $f^{r}(x)=f(x) \varphi^{r}(x)$, where $f \in C^{\infty} \cap C_{a}^{p}$, it easily follows that

$$
f_{|y| \leq t}\left|f^{r}(x+y)-f^{r}(x)\right| d y \leq C\left\|\varphi^{r}\right\|_{\infty} E f(x, t)+t\left\|\nabla \varphi^{r}\right\|_{\infty}|f(x)| ;
$$

thus, given $\varepsilon$, if $T^{1-a}\|f\|_{p} \leq \varepsilon,\left\|G_{a} f(\cdot, T)\right\|_{p} \leq \varepsilon$, and $r$ is small enough, we have

$$
\begin{aligned}
\left\|G_{a}\left(f-f^{r}\right)\right\|_{p} \leq & C\left\|G_{a} f(\cdot, T)\right\|_{p}+C T^{1-a}\|f\|_{p} \\
& +C\left\|\int_{T}^{\infty}\left|f-f^{r}\right| * \chi_{t}(\cdot) t^{-a-1} d t\right\|_{p} \\
\leq & C \varepsilon+C T^{-a}\left\|f-f^{r}\right\|_{p} \leq C \varepsilon .
\end{aligned}
$$

Hence, $C_{0}^{\infty}$ is dense in $F_{a}^{p}$, which together with Theorem 4 implies the density of $C^{\infty} \cap F_{a}^{p}$ in $F_{a}^{p}$ for all $a>0$. Finally, the density of $C_{0}^{\infty}$ in these $F_{a}^{p}$ follows as before.

If $a>n / p$ functions in $C_{a}^{p}$ are continuous [8, p. 74], whereas if $a \leq n / p$ they have a considerable degree of integrability.

PROPOSITION 2. If $1 \leq p<n / a, q=n p / n-a p$ and $f \in C_{a}^{p}$,

$$
\left(f_{Q}\left|f-P_{Q} f\right|^{q}\right)^{1 / q} \leq C|Q|^{a / n}\left(f_{Q}\left(G_{a} f\right)^{p}\right)^{1 / p}
$$

for any cube $Q$; if $p>1, a=n / p$ and $p^{\prime}=p / p-1$, there are constants $C, \beta$ such that for any cube $Q$

$$
f_{Q} \exp \left(\beta\left(\left|f-P_{Q} f\right| /\left\|G_{a} f \chi_{Q}\right\|_{p}\right)^{p^{\prime}}\right) \leq C .
$$

This result, essentially proved in [8, Lemma 4.2$]$ also follows easily by the Sobolev and Trudinger inequalities for Riesz potentials $[\mathbf{1 1}, \mathbf{1 6}]$ from the next theorem.

THEOREM 5. If $0<r \leq 1, a \leq n / p$ and $f \in C_{a}^{p}$, then for any cube $Q$ and a.e. $y \in Q$,

$$
\left|f(y)-P_{Q} f(y)\right|^{r} \leq C I_{a r}\left(G_{a} f \chi_{4 Q}\right)^{r}(y) .
$$

PROOF. Denoting by $B_{y, s}$ the ball with center $y$ and side $s$, and by $S_{n-1}$ the unit sphere in $\mathbf{R}^{n}$, an easy modification of (8) together with (6), polar coordinates and Fubini's theorem give

$$
\begin{aligned}
\left|f(y)-P_{Q} f(y)\right|^{r} & \leq C \int_{0}^{t} E f(y, s)^{r} d s / s \\
& \leq C \int_{0}^{t}\left(f_{B_{y, s}} E f(z, s)^{r} d z\right) d s / s \\
& \leq C \int_{0}^{2 t} s^{a r} f_{B_{y, s}} G_{a} f(z)^{r} d z d s / s \\
& =C \int_{0}^{2 t} s^{a z-n} \int_{0}^{s} \int_{S_{n-1}} G_{a} f\left(x+u y^{\prime}\right)^{r} u^{n-1} d u d y^{\prime} d s / s \\
& \leq C \int_{0}^{2 t} \int_{S_{n-1}} u^{a r-n} G_{a} f\left(x+u y^{\prime}\right)^{r} u^{n-1} d y^{\prime} d u \\
& \leq C I_{a r}\left(G_{a} f \chi_{4 Q}\right)^{r}(y) .
\end{aligned}
$$


Observe that since $I_{a} \sim J_{a}$ near $0,\left|f(y)-P_{Q} f(y)\right| \leq C J_{a}\left(G_{a} f \chi_{4 Q}\right)(y)$; also $\left\|P_{Q} f \chi_{Q}\right\|_{\infty} \leq C|f|_{Q}$ tends to 0 if $|Q|$ tends to $\infty$, and hence, $|f| \leq C I_{a}\left(G_{a} f\right)$ a.e. in $\mathbf{R}^{n}, 0<a<n / p$. Furthermore, if $1 \leq s<q$, (11) implies $E_{s} f(x, t) \leq$ $C t^{a}\left(M\left(G_{a} f\right)^{r}(x)\right)^{1 / r}$ for some $r<p$; therefore

$$
\left\|\sup _{t} t^{-a} E_{s} f(\cdot, t)\right\|_{p} \sim\left\|G_{a} f\right\|_{p}
$$

which for the same $s$ and $k \geq m$ extends to [8, p. 27]

$$
\sup _{t} t^{-a} E_{s}^{k} f(x, t) \sim \sup _{t} t^{-a} E_{s} f(x, t) .
$$

Finally we note that since ||$f(x+y)|-| f(x)|| \leq|f(x+y)-f(x)|$, if $f \in C_{a}^{p}$ or $F_{a}^{p}, 0<a<1$, so does $|f|$ and $\||f|\|_{a, p} \leq\|f\|_{a, p}$.

4. Tangential boundary values. We derive now Theorems 1 and 2 from pointwise estimates for the corresponding tangential maximal functions.

PROOF OF THEOREM 1. If $f \in F_{a}^{p}$ and $u(x, y)=P_{y} * f(x)$, define $T_{a, p} f(x)=$ $\sup \left\{|u(z, y)|:(z, y) \in D_{a, p}(x)\right\}$; we will show

$$
T_{a, p} f\left(x_{0}\right) \leq C\left(M f\left(x_{0}\right)+\left(M\left(G_{a} f\right)^{p}\left(x_{0}\right)\right)^{1 / p}\right) ;
$$

obviously, (14) implies that $\left|\left\{T_{a, p} f>t\right\}\right| \leq C\left(\|f\|_{a, p} / t\right)^{p}$, and standard arguments give then Theorem 1.

Suppose $x_{0}=0$; if $(x, y) \in D_{a, p}(0)$ and $Q=Q_{0,2|x|}$, we have

$$
|u(x, y)|=\left|\left(\int_{Q}+\int_{c_{Q}}\right) f(z) P_{y}(x-z) d z\right|=\mathrm{I}+\mathrm{II}
$$

if $z \in{ }^{c} Q,|z-x| \geq|z| / 2$ and $P_{y}(x-z) \leq P_{y}(z / 2)$; thus,

$$
\mathrm{II} \leq \int_{\mathbf{R}^{n}}|f(z)| P_{y}(z / 2) d z \leq C M f(0) .
$$

Next, by (4),

$$
\begin{aligned}
\mathrm{I} & \leq \int_{Q}\left|f(z)-P_{Q} f(z)\right| P_{y}(x-z) d z+\int_{Q}\left|P_{Q} f(z)\right| P_{y}(x-z) d z \\
& \leq \mathrm{III}+C M f(0) \int_{Q} P_{y}(x-z) d z \leq \mathrm{III}+C M f(0) .
\end{aligned}
$$

If $a<n / p, q=n p / n-a p$ and $q^{\prime}=q / q-1$, Hölder's inequality and Proposition 2 give

$$
\begin{aligned}
\mathrm{III} & \leq\left\|P_{y}\right\|_{q^{\prime}}\left(\int_{Q}\left|f-P_{Q} f\right|^{q}\right)^{1 / q} \\
& \leq C y^{-n / q}|x|^{a+n / q}|x|^{-a}\left(f_{Q}\left|P_{Q} f\right|^{q}\right)^{1 / q} \\
& \leq C y^{-n / q}|x|^{n / p}\left(M\left(G_{a} f\right)^{p}(0)\right)^{1 / p} \\
& \leq C\left(M\left(G_{a} f\right)^{p}(0)\right)^{1 / p},
\end{aligned}
$$

since $|x|<y^{p / q}$; thus, (14) is proved in this case. 
If $a=n / p, p>1$ and $p^{\prime}=p / p-1$, we will use an Orlicz space version of Hölder's inequality: if $\phi(t)=t(\log (1+t))^{1 / p^{\prime}}$ and $\Psi$ is its conjugate Orlicz function, then $\boldsymbol{\Psi}(t) \leq C e^{\alpha t^{p^{\prime}}}$ for appropriate $C$ and $\alpha$, and therefore [18, p. 171]

$$
\left|f_{Q} g h d z\right| \leq\|g\|_{\phi} \max \left(1, f_{Q} \Psi(h) d z\right) \leq C\|g\|_{\phi} f_{Q} e^{\alpha|h|^{p^{\prime}}} d z
$$

where $\|g\|_{\phi}$ denotes the $\phi$-Orlicz norm of $g \chi_{Q}$ with respect to $d z /|Q|$. This inequality and Proposition 2 imply

$$
\begin{aligned}
\mathrm{III} & \leq C\left\|G_{a} f \chi_{Q}\right\|_{p}|Q| f_{Q} P_{y}(x-z) \frac{\left|f(z)-P_{Q} f(z)\right|}{\left\|G_{a} f \chi_{Q}\right\|_{p}} d z \\
& \leq C|x|^{n+n / p}\left(M\left(G_{a} f\right)^{p}(0)\right)^{1 / p}\left\|P_{y}(x-\cdot)\right\|_{\phi} \int_{Q} \exp \left(\beta\left(\frac{\left|f-P_{Q} f\right|}{\left\|G_{a} f \chi_{Q}\right\|_{p}}\right)^{p^{\prime}}\right) d z \\
& \leq C|x|^{n+n / p}\left(M\left(G_{a} f\right)^{p}(0)\right)^{1 / p}\left\|P_{y}(x-\cdot)\right\|_{\phi} .
\end{aligned}
$$

But $\left\|P_{y}(x-\cdot)\right\|_{\phi}=\inf \left\{t: f_{Q} \phi\left(P_{y}(x-z) / t\right) d z \leq 1\right\}[\mathbf{1 8}$, p. 173]; thus, if $T=$ $C_{0}(\log 1 / y)^{1 / p^{\prime}} /|Q|, C_{0}$ to be fixed later, then $T \geq C_{0} / 2^{n}|x|^{n}(\log 1 / y)^{p / p^{\prime}} \geq C_{0} 2^{-n}$, for $(x, y) \in D_{a, p}(0)$, and therefore,

$$
\begin{array}{r}
f_{Q}\left(P_{y}(x-z) / T\right)\left(\log \left(1+P_{y}(x-z) / T\right)^{1 / p^{\prime}} d z\right. \\
\quad \leq \int_{2 Q} P_{y}(z)\left(\log \left(1+c_{n} y^{-n}\right)\right)^{1 / p^{\prime}} d z / T|Q| \\
\quad \leq C\left((\log 1 / y)^{1 / p^{\prime}} / T|Q|\right) \int_{2 Q} P_{y}(z) d z \leq 1
\end{array}
$$

for an appropriate $C_{0}$. Hence, $\left\|P_{y}(x-\cdot)\right\|_{\phi} \leq T$ and we have

$$
\begin{aligned}
\mathrm{III} & \leq C|x|^{n+n / p}|x|^{-n}(\log 1 / y)^{1 / p^{\prime}}\left(M\left(G_{a} f\right)^{p}(0)\right)^{1 / p} \\
& \leq C\left(M\left(G_{a} f\right)^{p}(0)\right)^{1 / p} .
\end{aligned}
$$

PROOF OF THEOREM 2. If $1<p<n / a$, Theorem 5 and Lemma 3 imply that $M f(x) \leq C I_{a} G_{a} f(x)$, and it easily follows that $f$ can be redefined in a zero measure set so that the complement of the Lebesgue set of the new $f$ has zero $R_{a, p}$, and hence, $B_{a, p}$ capacity; clearly this implies nontangential convergence $B_{a, p}$-a.e. When $p=1$, the embeddings $F_{a}^{1} \subset F_{a-n / p^{\prime}}^{p} \subset L_{a-n / p^{\prime}-e}^{p}, 1<p<n / n-a, e>0$ $\left[8\right.$, pp. 72 and 58] tell us that any $f \in F_{a}^{1}$ can be redefined in a zero measure so that the complement of its Lebesgue set has zero $B_{a-n / p^{\prime}-e, p}$ capacity and hence, zero $H^{(n-a) p+p e}$ Hausdorff measure [12]. Thus, for any $\varepsilon>0$, we have nontangential convergence of $P_{y} * f$ for all $x$ outside a set of zero $H^{n-a+\varepsilon}$ Hausdorff measure.

Next, if $0<b<a$, fix $x_{0}=0$ and $(x, y) \in D_{b, p}(0)$. Proceeding as in the proof of Theorem 1 , we obtain $|u(x, y)| \leq \mathrm{III}+C M f(0)$, and setting $r=n p / n-b p$, Hölder's 
inequality and Theorem 5 imply

$$
\begin{aligned}
\text { III } & \leq C y^{-n / r}|x|^{a+n / r}|x|^{-a}\left(f_{Q}\left|f-P_{Q} f\right|^{q}\right)^{1 / q} \\
& \leq C y^{-n / r}|x|^{n / p+a-b}\left(f_{Q}\left(G_{a} f\right)^{p}\right)^{1 / p} \leq C|x|^{a-b}\left(f_{Q}\left(G_{a} f\right)^{p}\right)^{1 / p} \\
& \leq C\left(M_{(a-b) p}\left(G_{a} f\right)^{p}(0)\right)^{1 / p},
\end{aligned}
$$

since $|x| \leq y^{p / r}$. Also, $M f \leq C I_{a} G_{a} f$ and therefore

$$
T_{b, p} f(0) \leq C\left(M_{(a-b) p}\left(G_{a} f\right)^{p}(0)\right)^{1 / p}+C I_{a}\left(G_{a} f\right)(0),
$$

which by Lemmas 1 and 2 gives

$$
\begin{aligned}
H_{\infty}^{n-(a-b) p}\left(\left\{T_{b, p} f>t\right\}\right) \leq & H_{\infty}^{n-(a-b) p}\left(\left\{M_{(a-b) p}\left(G_{a} f\right)^{p}>t^{p} / C\right\}\right) \\
& +H_{\infty}^{n-(a-b) p}\left(\left\{I_{a} G_{a} f>t / C\right\}\right) \\
\leq & C\left(\left\|G_{a} f\right\|_{p} / t\right)^{p}+C\left(\left\|G_{a} f\right\|_{p} / t\right)^{p(n-(a-b) p) / n-a p}
\end{aligned}
$$

and standard arguments finish now the proof of part (i).

In part (ii) we first divide $\mathbf{R}^{n}$ into a mesh of disjoint cubes of side $1 / 1000$. If $x$ is in such a cube $Q^{\prime}$,

$$
u(x, y)=\int P_{y}(x-z)\left(f \chi_{4 Q^{\prime}}(z)+f \chi_{c_{4 Q^{\prime}}}(z)\right) d z=u_{1}(x, y)+u_{2}(x, y),
$$

and since $\left|u_{2}(x, y)\right| \leq C y^{1 / p^{\prime}}\|f\|_{p}$ tends to 0 with $y$ uniformly in $2 Q^{\prime}$, it is enough to study the convergence of $u_{1}$. Fix now $x_{0}=0,(x, y) \in D_{n / r, r}(0), p<r<\infty$ and $Q=Q_{0,2|x|}$, and assume $0 \in Q^{\prime}$ with $Q^{\prime}$ in the above mesh, and $y$ small enough so that side $Q \leq 1 / 1000$. Using again the Orlicz space version of Hölder's inequality, this time with the function $\phi(t)=t(\log (1+t))^{1 / r^{\prime}}$, we obtain as in Theorem 1

$$
\begin{aligned}
\left|u_{1}(x, y)\right| & \leq \mathrm{III}+C M\left(f \chi_{4 Q^{\prime}}\right)(0) \\
& \leq C(\log 1 / y)^{1 / r^{\prime}}\left\|G_{n / p} f \chi_{Q}\right\|_{p}+C M\left(f \chi_{4 Q^{\prime}}\right)(0) ;
\end{aligned}
$$

now, Theorem 5, Lemma 3, and (4) give

$$
\begin{aligned}
M\left(f \chi_{4 Q^{\prime}}\right)(0) & \leq C M\left(I_{n / p}\left(G_{n / p} f \chi_{8 Q^{\prime}}\right)(0)+C|f|_{8 Q^{\prime}}\right. \\
& \leq C I_{n / p}\left(G_{n / p} f \chi_{8 Q^{\prime}}\right)(0)+C J_{n / p}\left(|f|_{8 Q^{\prime}}\right)(0) \\
& \leq C J_{n / p}\left(G_{n / p} f+|f|\right)(0),
\end{aligned}
$$

and since $(x, y) \in D_{n / r, r}(0)$,

$$
\begin{aligned}
\left|u_{1}(x, y)\right| & \leq C|x|^{n / r}\left(\int_{Q}\left(G_{n / p} f\right)^{p}\right)^{1 / p}+C J_{n / p}\left(G_{n / p} f+|f|\right)(0) \\
& \leq C\left(M_{n-n p / r}\left(G_{n / p} f\right)^{p}(0)\right)^{1 / p}+C J_{n / p}\left(G_{n / p} f+|f|\right)(0) .
\end{aligned}
$$

Thus, defining $T_{s, t}^{\prime} f(x)=\sup \left\{\left|u_{1}(z, y)\right|:(z, y) \in D_{s, t}(x)\right\}$, we have

$$
\begin{aligned}
H_{\infty}^{n p / r}\left(\left\{T_{n / r, r}^{\prime} f>t\right\}\right) \leq & H_{\infty}^{n p / r}\left(\left\{M_{n-n p / r}\left(G_{n / p} f\right)^{p}>t^{p} / C\right\}\right) \\
& +H_{\infty}^{n p / r}\left(\left\{J_{n / p}\left(G_{n / p} f+|f|\right)>t / C\right\}\right) \\
= & \mathrm{I}+\mathrm{II},
\end{aligned}
$$


where $\mathrm{I} \leq C\left\|G_{n / p} f\right\|_{p}^{p} / t^{p}$, by Lemma 1 . Also, since

$$
J_{n / p}\left(G_{n / p} f+|f|\right)=J_{n / p-e}\left(J_{e}\left(G_{n / p} f+|f|\right)\right) \leq I_{n / p-e} F,
$$

with $F=J_{e}\left(G_{n / p} f+|f|\right)$, then, if $e<n / r$, Lemma 2 implies that

$$
\mathrm{II} \leq C\left(\|F\|_{p} / t\right)^{n p / r e} \leq C\left(\|f\|_{n / p, p} / t\right)^{n p / r e} .
$$

Convergence inside $D_{n / r, r}(x)$ for $H^{n p / r}$-a.a. $x$ follows now by standard arguments from these estimates.

Finally, if $0 \leq b<n / p$ and $(x, y) \in D_{b, p}(0), \log 1 / y \leq C \log 1 /|x|$, and proceeding as before, we obtain

$$
\begin{aligned}
\left|u_{1}(x, y)\right| & \leq C\left((\log 1 /|x|)^{p-1} \int_{Q}\left(G_{n / p} f\right)^{p}\right)^{1 / p}+C M\left(f \chi_{4 Q^{\prime}}\right)(0) \\
& \leq C\left(M_{\varphi}\left(G_{n / p} f\right)^{p}(0)\right)^{1 / p}+C J_{n / p}\left(G_{n / p} f+|f|\right)(0),
\end{aligned}
$$

with $M_{\varphi}$ as in $\S 2$, and since $B_{n / p, p} \leq C H_{1 / 100}^{\varphi}[\mathbf{1 2}]$, it follows that

$$
\begin{aligned}
B_{n / p, p}\left(\left\{T_{b, p}^{\prime} f>t\right\}\right) \leq & C H_{1 / 100}^{\varphi}\left(\left\{M_{\varphi}\left(G_{n / p} f\right)^{p}>t^{p} / C\right\}\right) \\
& +B_{n / p, p}\left(\left\{J_{n / p}\left(G_{n / p} f+|f|\right)>t / C\right\}\right) \\
\leq & C\left(\|f\|_{n / p, p} / t\right)^{p}
\end{aligned}
$$

and again standard arguments can be used to finish the proof.

5. Strong $L^{p}$ estimates. The proof of Theorem 3 depends on a strong inequality for a certain capacity type set function associated to $C_{a}^{p}$ which we now define. Fix $a, b$ and $p$ with $0<b<a$ and $1 \leq p<\infty$, and for any $E \subset \mathbf{R}^{n}$ denote by $O_{a, p}^{b}(E)$ the set $\left\{g \in C_{b}^{p}: g \geq 0, J_{a-b} g \geq \chi_{E}\right\}$; we define then

$$
U_{a, p}^{b}(E)=\inf \left\{\left(\|g\|_{b, p}\right)^{p}: g \in O_{a, p}^{b}(E)\right\} .
$$

Obviously, $U_{a, p}^{b}(E) \leq U_{a, p}^{b}\left(E^{\prime}\right)$ if $E \subset E^{\prime}$ and $U_{a, p}^{b}(E \cup F) \leq C\left(U_{a, p}^{b}(E)+U_{a, p}^{b}(F)\right)$; furthermore, by Theorem 4 , if $g \in C_{b}^{p}$,

$$
U_{a, p}^{b}\left(\left\{J_{a-b}(g)>t\right\}\right) \leq\left(\|g\|_{b, p} / t\right)^{p} \leq C\left(\left\|J_{a-b} g\right\|_{a, p} / t\right)^{p} ;
$$

it can also be proved that $U_{a, p}^{b} \sim U_{a, p}^{b^{\prime}}$ if $0<b, b^{\prime}<a$ and that $R_{a, p} \leq C U_{a, p}^{b}$, $a<n / p$; since we clearly have $U_{a, p}^{b} \leq C B_{a, p}$, it follows that $R_{a, p}, B_{a, p}$ and $U_{a, p}^{b}$ have the same zero sets when $a<n / p$ (Lemma 3 and (15) imply that any $f \in F_{a}^{p}$ can be modified in a set of measure zero so that the complement of its Lebesgue set has zero $U_{a, p}^{b}$ "capacity", and hence zero $B_{a, p}$ capacity if $\left.1<p<\infty\right)$.

$U_{a, p}^{b}$ satisfies the following strong type inequality.

THEOREM 6. If $0<b<a, 1 \leq p<\infty$, and $g \in C_{b}^{p}, g \geq 0$, then

$$
\int_{0}^{\infty} s^{p-1} U_{a, p}^{b}\left(\left\{J_{a-b} g>s\right\}\right) d s \leq C\left(\left\|J_{a-b} g\right\|_{a, p}\right)^{p} .
$$

Once this is proved, Theorem 3 is deduced as in [14]: given $A \subset \mathbf{R}^{n}$ and $B \subset$ $\mathbf{R}_{+}^{n+1}$ set $S(A)=\mathbf{R}_{+}^{n+1}-\bigcup\{C(x): x \notin A\}$, with $C(x)=\left\{(z, y) \in \mathbf{R}_{+}^{n+1}:|z-x| \leq\right.$ $y\}$, and $J(B)=\left\{x \in \mathbf{R}^{n}: B \cap D_{a, p}(x) \neq \varnothing\right\}$; then, if $g \in O_{a, p}^{b}(E)$, and $g^{\prime}=J_{a-b} g$, $J(S(E)) \subset\left\{T_{a, p} g^{\prime} \geq C_{0}\right\}$ for some numerical $C_{0}$, and the weak inequalities of 
Theorem 1 give $|J(S(E))| \leq C\left(\|g\|_{b, p}\right)^{p}$. Thus, taking inf in $O_{a, p}^{b}(E)$, we obtain $|J(S(E))| \leq C U_{a, p}^{b}(E)$. Now, if $f \in C_{a}^{p}$,

$$
\left\{T_{a, p} f>s\right\} \subset J(S(\{N f>s / C\})),
$$

where $N f(x)=\sup \{|u(x, y)|: y>0\}$; writing $f=J_{a-b}(g), g \in C_{b}^{p}, 0<b<1$, we have $N f(x) \leq J_{a-b}(N(|g|))(x)$, but

$$
\int|g|\left(x^{\prime}-z\right) P_{y}(z) d z=\int_{|z| \leq 2}+\int_{|z|>2}=\mathrm{I}+\mathrm{II},
$$

and standard arguments give $\mathrm{I} \leq C m(|g|)\left(x^{\prime}\right)$, which since $|g|=J_{b-e} h, h \in C_{e}^{p}$, implies I $\leq C m\left(J_{b-e}|h|\right)\left(x^{\prime}\right) \leq C J_{b-e}|h|\left(x^{\prime}\right)$, by Lemma 3. Also, if $y \leq 1$ and $|z| \geq 2, P_{y}(z) \leq C /(1+|z|)^{n+1}=Q(z)$ and II $\leq Q *|g|\left(x^{\prime}\right)$, which belongs to $C_{b}^{p}$. Therefore $N f \leq C\left(J_{a-e}|h|+J_{a-b}(Q *|g|)\right)$, and since $\|Q *|g|\|_{b, p} \leq C\|f\|_{a, p}$ and $\||h|\|_{e, p} \leq C\|f\|_{a, p}$, Theorem 6 gives

$$
\begin{aligned}
\left\|T_{a, p} f\right\|_{p}^{p} & =p \int_{0}^{\infty} s^{p-1}\left|\left\{T_{a, p} f>s\right\}\right| d s \\
& \leq C \int_{0}^{\infty} s^{p-1} U_{a, p}^{b}(\{N f>s / C\}) d s \\
& \leq C \int_{0}^{\infty} s^{p-1}\left(U_{a, p}^{b}\left(\left\{J_{a-b}\left(J_{b-e}|h|\right)>s\right\}\right)\right) \\
& \quad+\left(U_{a, p}^{b}\left(\left\{J_{a-b}(Q *|g|)>s\right\}\right)\right) d s \\
& \leq C\left(\||h|\|_{e, p}^{p}+\|Q *|g|\|_{b, p}^{p}\right) \leq C\|f\|_{a, p}^{p} .
\end{aligned}
$$

Our proof of Theorem 6 is an adaptation and simplification of the one given by Dahlberg in $[\mathbf{7}]$; we need a preliminary lemma.

LEMMA 4. Let $h$ be a $C^{\infty}$ function with $h(t)=0$ if $t<0$, and $\left|t^{j-1} h^{(j)}(t)\right| \leq A$, $0 \leq j \leq m+1, m=[a]$. Then if $f=J_{a-b} g$, with $g \in C_{b}^{p}$, and $g \geq 0, h(f) \in C_{a}^{p}$ and $\|h(f)\|_{a, p} \leq C\|f\|_{a, p}$.

Proof. We will estimate $G_{a} h(f)(x, 1)$ using the centered version of $E$ (see $\S 3$ ). Fix $x \in \mathbf{R}^{n}$ and write $Q$ for $Q_{x, t}$, where we assume $t \leq 1$. Writing $P_{Q} f(y)=$ $\sum_{|\gamma| \leq m} c_{\gamma}(t)(y-x)^{\gamma} / \gamma !$, define

$$
R(y)=f(x)+\sum_{0<|\gamma| \leq m} c_{\gamma}(t)(y-x)^{\gamma} / \gamma !
$$

by (4) and Lemma 3 we have

$$
\begin{aligned}
|R(y)| & \geq f(x)-C \sum_{0<|\gamma| \leq m} f_{Q}(|y-x| / t)^{|\gamma|} \\
& \geq f(x)\left(1-C \sum_{0<|\gamma| \leq m}(|y-x| / t)^{|\gamma|}\right) ;
\end{aligned}
$$

thus, if $|x-y|<\varepsilon t$ with $\varepsilon$ small enough, $R(y)>C f(x)$. Considering now the polynomial

$$
S(y)=\sum_{0}^{m} h^{(j)}(R(x))(R(y)-R(x))^{j} / j !
$$


Taylor's formula and (8) give

$$
\begin{aligned}
|h(f(y))-S(y)| \leq & |h(f(y))-h(R(y))|+|h(R(y))-S(y)| \\
\leq & A|f(y)-R(y)|+C|R(y)-R(x)|^{m+1} \\
& \times\left|h^{(m+1)}((1-\theta) R(x)+\theta R(y))\right| \\
\leq & C\left(\left|f(y)-P_{Q} f(y)\right|+t^{a} G_{a} f(x)\right. \\
& \left.\quad+|R(y)-R(x)|^{m+1} / f(x)^{m}\right) .
\end{aligned}
$$

Set now $T=\left(f(x) / G_{a} f(x)\right)^{1 / a}$ and suppose $T \leq 1$; since

$$
(R(y)-R(x))^{m+1}=\sum_{j=m}^{m(m+1)} \sum_{|\gamma|=j} c_{\gamma}(y-x)^{\gamma}
$$

where $c_{\gamma}$ equals the sum of all terms $c_{\gamma_{1}}(t) \cdots c_{\gamma_{m+1}}(t)$ with $\gamma_{1}+\cdots+\gamma_{m+1}=\gamma$, then (7) and (4) imply for $0<t \leq T$ that

$$
\begin{aligned}
\left|c_{\gamma_{i}}(t)\right| & \leq C T^{-\left|\gamma_{i}\right|} f_{Q_{x, T}}+C T^{a-\left|\gamma_{i}\right|} G_{a} f(x) \\
& \leq C T^{-\left|\gamma_{i}\right|}\left(f(x)+T^{a} G_{a} f(x)\right) \leq C T^{-\left|\gamma_{i}\right|} f(x),
\end{aligned}
$$

if $\left|\gamma_{i}\right|<a$, or

$$
\begin{aligned}
\left|c_{\gamma_{i}}(t)\right| & \leq C T^{-\left|\gamma_{i}\right|} f(x)+C \log (T / t) G_{a} f(x) \\
& \leq C T^{-\left|\gamma_{i}\right|} \log (T / t) f(x)
\end{aligned}
$$

if $\left|\gamma_{i}\right|=a$. In any case,

$$
|R(y)-R(x)|^{m+1} \leq C f(x)^{m+1}(\log e T / t)^{m+1} \sum_{m+1}^{m(m+1)}(t / T)^{j},
$$

which implies

$$
\begin{aligned}
& E^{m(m+1)} h(f)(x, t) \\
& \quad \leq C\left(E f(x, t)+t^{a} G_{a} f(x)+f(x)(\log e T / t)^{m+1} \sum_{m+1}^{m(m+1)}(t / T)^{j}\right),
\end{aligned}
$$

and therefore

$$
\sup _{t \leq T} t^{-a} E^{m(m+1)} h(f)(x, t) \leq C\left(G_{a} f(x)+f(x) T^{-a}\right) \leq C G_{a} f(x) .
$$

If $T \leq t \leq 1$, then $E^{m(m+1)} h(f)(x, t) \leq C f_{Q} \leq C f(x)$, by (4) and Lemma 3, and we have

$$
\sup _{T \leq t \leq 1} t^{-a} E^{m(m+1)} h(f)(x, t) \leq C T^{-a} f(x) \leq C G_{a} f(x) .
$$

In the case $T>1$, then $f(x)>G_{a} f(x)$ and we estimate the coefficients $c_{\gamma_{i}}(t)$ as $\left|c_{\gamma_{i}}(t)\right| \leq C\left(f(x)+\log (e / t) G_{a} f(x)\right) \leq C \log (e / t) f(x)$, and replace (16) by

(18) $\sup _{t \leq 1} t^{-a} E^{m(m+1)} h(f)(x, t) \leq C\left(G_{a} f(x)+f(x) \sum_{m+1}^{m(m+1)} \sup _{t \leq 1} t^{j-a} \log (e / t)\right)$

$$
\leq C f(x) \text {. }
$$


Thus, (16), (17), and (18) yield for a.e. $x$

$$
G_{a} h(f)(x, 1) \leq C\left(G_{a} f(x)+f(x)\right)
$$

and, since $h(f) \leq A f$, we conclude that $\|h(f)\|_{a, p} \leq C\|f\|_{a, p}$.

To finish the proof of Theorem 6 fix $a, b, p$ and write $U$ instead of $U_{a, p}^{b}$. As in $[2$ or 7], if $h$ is a $C^{\infty}$ function with $h(t)=0$ if $t<0, h(t)=1$ if $t>1$, define for any integer $j h_{j}(t)=2^{j} h\left(2^{2-j} t-1\right)$ and $f_{j}=h_{j}(f)$. By Lemma $4 f_{j} \in C_{a}^{p}$, and since $f_{j}(x)=2^{j}$ if $f(x)>2^{j},(15)$ gives

$$
\int_{0}^{\infty} s^{p-1} U(\{f>s\}) d s \leq C \sum_{-\infty}^{\infty} 2^{j p} U\left(\left\{f_{j} \geq 2^{j}\right\}\right) \leq C \sum_{-\infty}^{\infty}\left(\left\|f_{j}\right\|_{a, p}\right)^{p} .
$$

Now, the $h_{j}^{\prime}$ have disjoint support and are uniformly bounded; therefore,

$$
\begin{aligned}
\sum_{-\infty}^{\infty}\left(f_{j}(x)\right)^{p} & =\sum_{-\infty}^{\infty}\left(\int_{0}^{f(x)} h_{j}^{\prime}(s) d s\right)^{p} \\
& \leq f(x)^{p-1} \sum_{-\infty}^{\infty} \int_{0}^{f(x)}\left|h_{j}^{\prime}(s)\right| d s \leq C f(x)^{p}
\end{aligned}
$$

and $\sum\left\|f_{j}\right\|_{p}^{p} \leq C\|f\|_{p}^{p}$. Fix $x \in \mathbf{R}^{n}$ and denote $Q_{x, t}$ as $Q$; if $t \leq 1$, (4) and Lemma 3 give $f(x) \leq\left|f(x)-P_{Q} f(x)\right|+C f_{Q} \leq C t^{a} G_{a} f(x)+C^{\prime} f(y)$ for any $y \in Q$; thus,

$$
f(y) \geq\left(f(x)-C t^{a} G_{a} f(x)\right) / C^{\prime}>f(x) / 2 C^{\prime}
$$

if $t \leq T=\left(\varepsilon f(x) / G_{a} f(x)\right)^{1 / a}$ with $\varepsilon$ small enough. Now $C^{\prime} \sim 2^{K}$ for some $K$ independent of $f$ or $x$, and, when $f(x)>2^{j+K+1}, f(y)>2^{j}$ on $Q$ and $f_{j}(y)=2^{j}$. Hence, using again the centered version of $E$,

$$
E f_{j}(x, t) \leq f_{Q}\left|f_{j}(y)-f_{j}(x)\right| d y=0 .
$$

If $t \geq \min (1, T)$, then $E f_{j}(x, t) \leq C\left(f_{j}\right)_{Q} \leq C 2^{j}=C f_{j}(x)$ and therefore

$$
G_{a} f_{j}(x, 1) \leq \sup _{t>\min (1, T)} t^{-a} E f_{j}(x, t) \leq C\left(f_{j}(x)+T^{-a} f_{j}(x)\right),
$$

and (20) gives ( $\sim$ means the index set equals the preceding one)

$$
\begin{aligned}
\sum_{f(x)>2^{j+K+1}} G_{a} f_{j}(x, 1)^{p} & \leq C \sum_{\sim}\left(f_{j}(x)^{p}+T^{-a p} f_{j}(x)^{p}\right) \\
& \leq C\left(f(x)^{p}+G_{a} f(x)^{p}\right) .
\end{aligned}
$$

Suppose next $f(x)<2^{j-3}$; if $t \leq 1$ and we set as before $R(y)=f(x)+P_{Q} f(x)-$ $c_{0}(t)$,

$$
|R(y)| \leq f(x)+C \sum_{0<|\gamma| \leq m} f_{Q}(|y-x| / t)^{|\gamma|} \leq f(x)\left(1+C \sum_{\sim}(|y-x| / t)^{|\gamma|}\right)
$$


if $|x-y| \leq \varepsilon t$ with ' $\varepsilon$ small enough. Hence $h_{j}(R(y))=0$ and, setting $S=\{y \in$ $\left.Q: f(y)>2^{j-2}\right\},(4)$ implies

$$
\begin{aligned}
E f_{j}(x, \varepsilon t) & \leq C f_{Q} f_{j}(y) d y=C t^{-n} \int_{S} h_{j}(f(y)) d y \\
& \leq C t^{-n} \int_{S}\left|h_{j}(f(y))-h_{j}(R(y))\right| d y \\
& \leq C\left(f_{Q}|f-R|^{s}\right)^{1 / s}\left(t^{-n}|S|\right)^{1-1 / s} \\
& \leq C\left(E_{s} f(x, t)+t^{a} G_{a} f(x)\right)\left(2^{-j} f(x)\right)^{1-1 / s},
\end{aligned}
$$

for, by Lemma $3,|S| \leq C 2^{-j} \int_{Q} f d z \leq C 2^{-j} t^{n} f(x)$. Thus,

$$
G_{a} f_{j}(x, 1) \leq C\left(\sup _{t} t^{-a} E_{s} f(x, t)+G_{a} f(x)\right)\left(2^{-j} f(x)\right)^{1-1 / s},
$$

and since $\sum_{f(x)<2^{j-3}} 2^{-j p(1-1 / s)} \leq C f(x)^{-p(1-1 / s)}$,

$$
\sum_{f(x)<2^{j-3}} G_{a} f_{j}(x, 1)^{p} \leq C\left(\sup _{t} t^{-a} E_{s} f(x, t)\right) .
$$

By (19), we estimate the remaining $K+4 f_{j}$ as $G_{a} f_{j}(x, 1) \leq C\left(G_{a} f(x)+f(x)\right)$, which with (21) and (22) gives

$$
\sum_{-\infty}^{\infty} G_{a} f_{j}(x, 1)^{p} \leq C\left(\left(\sup _{t} t^{-a} E_{s} f(x, t)\right)^{p}+f(x)^{p}\right) ;
$$

taking now (12) and (20) into account, we obtain

$$
\sum_{-\infty}^{\infty}\left\|G_{a} f_{j}(\cdot, 1)\right\|_{p}^{p} \leq C\left(\left\|G_{a} f\right\|_{p}^{p}+\|f\|_{p}^{p}\right) \leq C\|f\|_{a, p}
$$

and the proof of Theorem 6 is finished.

6. Further remarks. We discuss here the imbeddings of the Triebel-Lizorkin spaces in $F_{a}^{p}$. These spaces are usually defined as follows [17]: let $\psi$ be a function in Schwartz's class $S$ such that $\Psi=\hat{\psi} \geq 0$ and $\operatorname{supp} \Psi \subset\{z: 1 / 2 \leq|z| \leq 2\}$, and set $\psi_{t}(z)=t^{-n} \psi(z / t)$; then $F_{a}^{p, q}, a>0,1 \leq p, q \leq \infty$ is the space of those $L^{p}$ functions such that

$$
D_{a, p} f(x)=\left(\int_{0}^{\infty}\left(t^{-a}\left|f * \psi_{t}(x)\right|\right)^{q} d t / t\right)^{1 / q}
$$

is in $L^{p}$. With the norm $\|f\|_{a, p, q}=\|f\|_{p}+\left\|D_{a, q} f\right\|_{p}, F_{a}^{p, q}$ becomes a Banach space, and as mentioned before, if $1<p<\infty, F_{a}^{p, 2}=L_{a}^{p}$, and $F_{a}^{1,2}=J_{a}\left(h^{1}\right)$.

The extension of Theorems 1,2 and 3 to the $F_{a}^{p, q}$ is a consequence of

PROPOSITION 3. If $1 \leq p, q<\infty, F_{a}^{p, q}$ is continuously imbedded in $F_{a}^{p}$.

PROOF. If $0<a<1, f \in F_{a}^{p, q}$ iff

$$
S_{a, q} f(x)=\left(\int_{0}^{\infty}\left(t^{-a} \int_{|y| \leq 1}|f(x+t y)-f(x)| d y\right)^{q} d t / t\right)^{1 / q}
$$


is in $L^{p}$ and $\left\|D_{a, q} f\right\|_{p} \sim\left\|S_{a, q} f\right\|_{p}\left[\mathbf{1 7}\right.$, p. 108]. But then $G_{a} f(x) \leq C S_{a, q} f(x)$ (see $\S 3)$ and therefore, $\|f\|_{a, p} \leq C\|f\|_{a, p, q}$. The general case is reduced to this one by Theorem 4 and the fact $\left[\mathbf{1 7}\right.$, p. 58] that the Bessel operator $J_{b}$ is an isomorphism between $F_{a}^{p, q}$ and $F_{a+b}^{p, q}$ (in fact it can be shown that $f \in F_{a}^{p, q}, 1 \leq p, q<\infty, a>0$ iff

$$
G_{a, q} f(x)=\left(\int_{0}^{\infty}\left(t^{-a} E f(x, t)\right)^{q} d t / t\right)^{1 / q}
$$

is in $L^{p}$, and $\left.\|f\|_{a, p, q} \sim\|f\|_{p}+\left\|G_{a, q}\right\|_{p}\right)$.

As a consequence, Theorems 1, 2, and 3 also hold for certain Besov spaces (see $[16,17]$ for their definition): indeed, if $1 \leq r \leq p, B_{a}^{p, r}$ is continuously imbedded in $F_{a}^{p, r}\left[\mathbf{1 7}\right.$, p. 47]. If $r>p$, the methods used here do not apply to $B_{a}^{p, r}$, although the embeddings $B_{a}^{p, r} \subset L_{a-\varepsilon}^{p}$ yield convergence of the Poisson integral of $f \in B_{a}^{p, r}$ inside any region $D_{a-\varepsilon, p}, \varepsilon>0$; since $C_{a}^{p} \subset L_{a-\varepsilon}^{p}$, the same is true of $C_{a}^{p}$.

\section{REFERENCES}

1. D. R. Adams, Quasiadditivity and sets of finite $L^{p}$ capacity, Pacific J. Math. 79 (1978), 283-291.

2. _ On the existence of capacitary strong type estimates in $\mathbf{R}^{n}$, Ark. Mat. 14 (1976), 125-140.

3. N. Aronszajn and K. T. Smith, Theory of Bessel potentials. I, Ann. Inst. Fourier 11 (1961), 385-475.

4. A. P. Calderón and R. Scott, Sobolev type inequalities for $p>0$, Studia Math. 62 (1978), 75-92.

5. A. P. Calderón and A. Zygmund, Local properties of solutions of elliptic partial differential equations, Studia Math. 20 (1961), 171-225.

6. L. Carleson, Selected problems on exceptional sets, Van Nostrand, 1967.

7. B. Dahlberg, Regularity properties of Riesz potentials, Indiana Univ. Math. J. 28 (1979), 257-268.

8. R. Devore and R. Sharpley, Maximal operators and smoothness, Mem. Amer. Math. Soc., No. 293 (1984).

9. J. R. Dorronsoro, A characterization of potential spaces, Proc. Amer. Math. Soc. 95 (1985), 21-31.

10. K. Hansson, Imbedding theorems of Sobolev type in potential theory, Math. Scand. 45 (1979), 77-102.

11. L. I. Hedberg, On certain convolution inequalities, Proc. Amer. Math. Soc. 36 (1972), 505-510.

12. N. G. Meyers, A theory of capacities for potentials of functions in Lebesgue spaces, Math. Scand. 26 (1970), 255-292.

13. Y. Mizuta, On the boundary limits of harmonic functions with gradient in $L^{p}$, Ann. Inst. Fourier (Grenoble) 34 (1984), 99-109.

14. A. Nagel, W. Rudin and J. Shapiro, Tangential boundary behavior of functions in Dirichlet type spaces, Ann. of Math. (2) 116 (1982), 331-360.

15. A. Nagel and E. M. Stein, On certain maximal functions and approach regions, Adv. in Math. 54 (1984), 83-106.

16. E. M. Stein, Singular integrals and differentiability properties of functions, Princeton Univ. Press, 1970.

17. H. Triebel, Theory of function spaces, Birkhäuser, 1983.

18. A. Zygmund, Trigonometric series, Cambridge Univ. Press, 1959.

División de matemáticas, facultad de Ciencias, Universidad Autónoma, 28049 MADRID, SPAIN 